\title{
Observation of Single-Spin Dirac Fermions at the Graphene/ Ferromagnet Interface
}

\author{
Dmitry Usachov, ${ }^{*}{ }^{\dagger}$ Alexander Fedorov, ${ }^{\dagger, \ddagger, \Phi l}$ Mikhail M. Otrokov, ${ }^{\S, \|}$ Alla Chikina, ${ }^{\dagger, \perp}$ Oleg Vilkov, ${ }^{\dagger}$
} Anatoly Petukhov, ${ }^{\dagger}$ Artem G. Rybkin, ${ }^{\dagger}$ Yury M. Koroteev, ${ }^{\S, \#}$ Evgueni V. Chulkov, ${ }^{\S, \|}$ Vera K. Adamchuk, ${ }^{\dagger}$ Alexander Grüneis, ${ }^{\ddagger}$ Clemens Laubschat, ${ }^{\perp}$ and Denis V. Vyalikh ${ }^{\dagger, \perp}$

${ }^{\dagger}$ St. Petersburg State University, 198504 St. Petersburg, Russia

${ }^{\ddagger}$ Physikalisches Institut, Universität zu Köln, Zülpicher Strasse 77, 50937 Köln, Germany

IIFW Dresden, P.O. Box 270116, D-01171 Dresden, Germany

${ }^{\S}$ Tomsk State University, Lenina Avenue 36, 634050 Tomsk, Russia

"Donostia International Physics Center (DIPC), Departamento de Fisica de Materiales and CFM-MPC UPV/EHU, 20080 San Sebastian, Spain

${ }^{\perp}$ Institute of Solid State Physics, Dresden University of Technology, D-01062 Dresden, Germany

${ }^{\#}$ Institute of Strength Physics and Materials Science of Siberian Branch Russian Academy of Sciences, pr. Akademicheskii, 2/4, 634021 Tomsk, Russia

Supporting Information

ABSTRACT: With the discovery and first characterization of graphene, its potential for spintronic applications was recognized immediately. Since then, an active field of research has developed trying to overcome the practical hurdles. One of the most severe challenges is to find appropriate interfaces between graphene and ferromagnetic layers, which are granting efficient injection of spin-polarized electrons. Here, we show that graphene grown under appropriate conditions on $\mathrm{Co}(0001)$ demonstrates perfect structural properties and simultaneously exhibits highly spin-polarized charge carriers. The latter was conclusively proven by observation of a singlespin Dirac cone near the Fermi level. This was accomplished experimentally using spin- and angle-resolved photoelectron spectroscopy, and theoretically with density functional calculations. Our results demonstrate that the graphene/ $\mathrm{Co}(0001)$ system represents an interesting candidate for applications in devices using the spin degree of freedom. KEYWORDS: Graphene, spin polarization, electronic structure, Dirac cone, spin-resolved ARPES

ectroscopy, and theoretically with density functional calculations.
presents an interesting candidate for applications in devices using
$\mathrm{T}^{\mathrm{r}}$ he most unique properties of the intrinsically twodimensional material graphene stem from its conelike dispersion of charge carries near the Fermi level, $E_{\mathrm{F}}$, where electron and hole pockets meet only at the $\overline{\mathbf{K}}$ points of the Brillouin zone. ${ }^{1}$ As a consequence, the carriers in graphene effectively behave as relativistic Dirac Fermions with zero effective mass. This is evidenced, for instance, by their outstanding mobility, which is observed in transport measurements. ${ }^{2}$ This behavior opens up significant possibilities for use in next generation electronics, ${ }^{3}$ where the spin-degree of freedom in particular might be used. ${ }^{4,5}$ However, graphene's unique electronic structure becomes strongly altered when brought into contact with ferromagnetic metals, which are used as spin-polarizing substrates. ${ }^{6,7}$ For any practical use it is therefore necessary to find an appropriate graphene/ferromagnet system where the remarkable properties of graphene and in

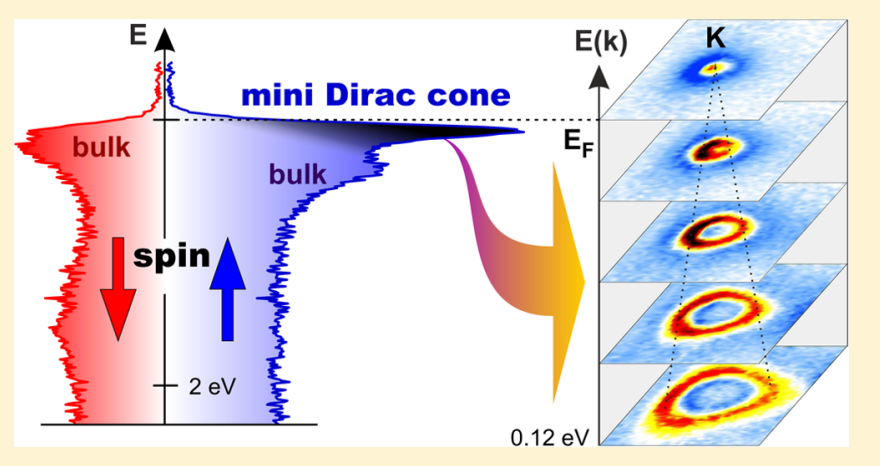

particular its conelike dispersion near the Fermi level are preserved.

Numerous studies of the electronic band structure of graphene/metal systems show that due to the strong interaction between $C 2 p_{z}$ and $d$ states of the metallic substrate, the $\pi$-states of graphene are essentially modified and largely shifted away from $E_{\mathrm{F}}$. For example, this has been shown for several metals, which are characterized by an open and chemically active d-shell, as is the case for $\mathrm{Ni}, \mathrm{Co}, \mathrm{Fe}, \mathrm{Rh}$, $\mathrm{Re}$, and $\mathrm{Ru}^{8-17}$ Only in the cases of weakly interacting substrates does the Dirac cone remain almost undisturbed. This is predicted theoretically ${ }^{7}$ and observed experimentally in graphene on $\mathrm{Au}, \mathrm{Cu}, \mathrm{Ir}, \mathrm{Pt}, \mathrm{Al}$, and on silicides, where the Dirac

Received: December 7, 2014

Revised: February 28, 2015

Published: March 3, 2015 
point is close to $E_{\mathrm{F}}{ }^{10,16,18-22}$ For the cases of magnetically interesting substrates like $\mathrm{Ni}(111)$ and $\mathrm{Co}(0001)$, the Dirac cone is modified in a very special way. It is split in several parts, which retain some similarities to the Dirac cone of freestanding graphene. The main part was identified in photoemission experiments as a gapless Dirac cone with its apex located at the binding energy of about $2.8 \mathrm{eV} .^{14}$ According to theoretical calculations the states close to the apex are notably mixed with metal 3d states. ${ }^{14,15,23}$ This mixing destroys the conical band above the energy of $2 \mathrm{eV}$. Thus, far, no ferromagnetic substrate is known where the electronic structure of free-standing graphene is widely conserved. Interestingly, theoretical considerations suggest that in spite of strong distortions of the linear dispersion of the $\pi$-states due to their hybridization with metal $\mathrm{d}$-states, a new spin-polarized conelike interface band near the Fermi level might exist. ${ }^{6,7,14,15,23-25}$ This offers an opportunity to obtain desired electronic and magnetic properties, and combine them in these composite structures.

Here, for the first time we experimentally demonstrate and theoretically support that such a Dirac-conelike feature exists in a graphene/ferromagnet system with its apex close to $E_{\mathrm{F}}$. Studying a perfectly oriented graphene/Co(0001) system by spin- and angle-resolved photoelectron spectroscopy (SRARPES), we found that this conelike band is formed by states with only one spin orientation. A system with such a property could act as a source of spin-polarized current, which is one of the main components of spintronic devices. The key aspect that gives rise to these properties is the perfect mutual orientation of the graphene layer and the $\mathrm{Co}(0001)$ substrate, which may be achieved following the appropriate growth protocol.

Structural Properties of Graphene/Co Interface. We explored the structural properties of a single graphene layer grown by chemical vapor deposition (CVD) on a layer of $\mathrm{Co}(0001)$ and discovered that the highly ordered graphene perfectly oriented with respect to the substrate could be grown only within a certain temperature range. As an example, in Figure 1 we show the results of low energy electron diffraction (LEED) and scanning tunneling microscopy (STM) characterization of graphene on $\mathrm{Co}(0001)$ prepared at the temperature of $560{ }^{\circ} \mathrm{C}$ (Figure 1a,c), as well as at $660{ }^{\circ} \mathrm{C}$ (Figure $1 \mathrm{~b}, \mathrm{~d}$ ). The latter is essentially quite high and close to its destruction temperature. The system prepared at the lower temperature is characterized in LEED by the arc-shaped reflexes (Figure 1a). This observation implies the presence of predominant misoriented domains, the existence of which has been established by other studies. ${ }^{11,13,14}$ The STM data taken from the system are consistent with the LEED results. They demonstrate a well-known moire pattern, which is intrinsic to the mismatched interface (Figure 1c).

Next, we looked closely at the LEED and STM data taken for the second system (Figure $1 \mathrm{~b}, \mathrm{~d}$ ), which was prepared at high temperature. In this case we can safely conclude that carbon atoms, packed into the graphene matrix, perfectly fit the lattice of the underlying cobalt layer. This is possible because the misfit between lattice parameters of free graphene and cobalt is less than $2 \%$. LEED shows a $(1 \times 1)$ structure and the STM image demonstrates a 3 -fold symmetry of the system, which indicates the nonequivalence of the two carbon sublattices $\mathrm{A}$ and B, depicted in inset in Figure 1d. This may correspond to the structure in which the atoms of one sublattice are located above Co atoms, while the others occupy hollow sites.

It should be noted that recently the formation of graphene/ $\mathrm{Co}(0001)$ interface with $(1 \times 1)$ LEED pattern was

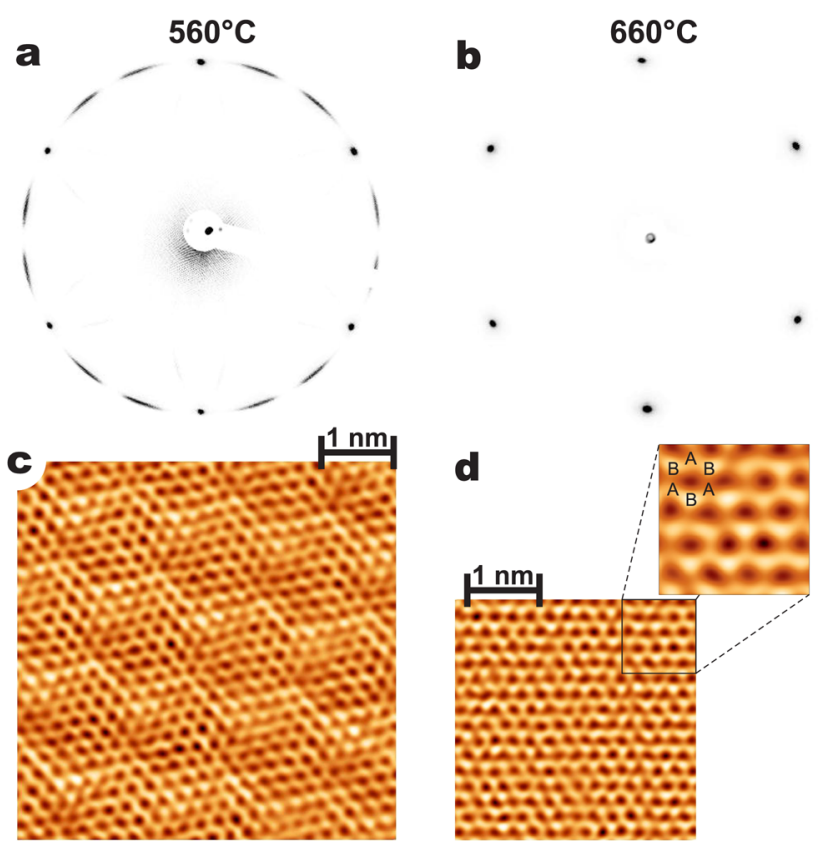

Figure 1. Graphene structure in real and reciprocal space. LEED patterns of graphene/Co(0001) for (a) misoriented graphene domains, and (b) perfectly oriented graphene. The electron energy was $70 \mathrm{eV}$. Respective STM images for (c) misoriented domain, rotated by $\sim 10^{\circ}$ relative to the $\mathrm{Co}(0001)$ lattice and (d) graphene aligned in a registry with the substrate. The horizontal direction is parallel to the $[11 \overline{2} 0]$ direction of cobalt. The inset shows a zoomed region manifesting nonequivalence of two sublattices. The synthesis temperature is shown above the images.

demonstrated on the Co film with the thickness of $3 \mathrm{~nm}$ at the growth temperature of $430{ }^{\circ} \mathrm{C} .{ }^{26}$ This suggests that the synthesis temperature may be not the decisive parameter, which ensures perfect graphene orientation. In our experiments, much thicker Co films were used (not thinner than $10 \mathrm{~nm}$ ), which was necessary for keeping the film integrity at the synthesis temperatures up to $660^{\circ} \mathrm{C}$. We suppose that thicker Co films may require annealing at higher temperature in order to reach good crystallization, which is important for graphene ordering. Also the type of hydrocarbon gas used in CVD may be important. Interestingly, despite the perfect graphene orientation no spin-polarized interface state was detected in ref 26 . This indicates certain structural differences between the systems formed at different conditions. The possible difference, which may be not reflected in the LEED pattern, is a presence of nonperiodic imperfections of the graphene/Co interface, formed at low temperature. In the case of $\mathrm{Ni}(111)$, it has been demonstrated that the quality of graphene is increasing with the synthesis temperature. ${ }^{27}$ Graphene grown at the temperatures of $400-450{ }^{\circ} \mathrm{C}$ is characterized by a high concentration of defects, while at $650{ }^{\circ} \mathrm{C}$ a high-quality lattice is formed. ${ }^{27}$ For this reason, we performed synthesis at high temperatures and used STM for proving the high graphene quality.

Electronic Structure Viewed by ARPES. The electronic structure of this perfectly oriented graphene on $\mathrm{Co}(0001)$ was next explored in detail by spin- and angle-resolved photoelectron spectroscopy. In Figure 2a, we present ARPES spectra taken at the $\overline{\mathbf{K}}$-point of the surface Brillouin zone (BZ) (the data at the $\bar{\Gamma}$-point are shown in Figure S3a in Supporting Information). Figure $2 \mathrm{~b}$ schematically shows the $\mathrm{BZ}$ of graphene and the direction of the ARPES measurements. 

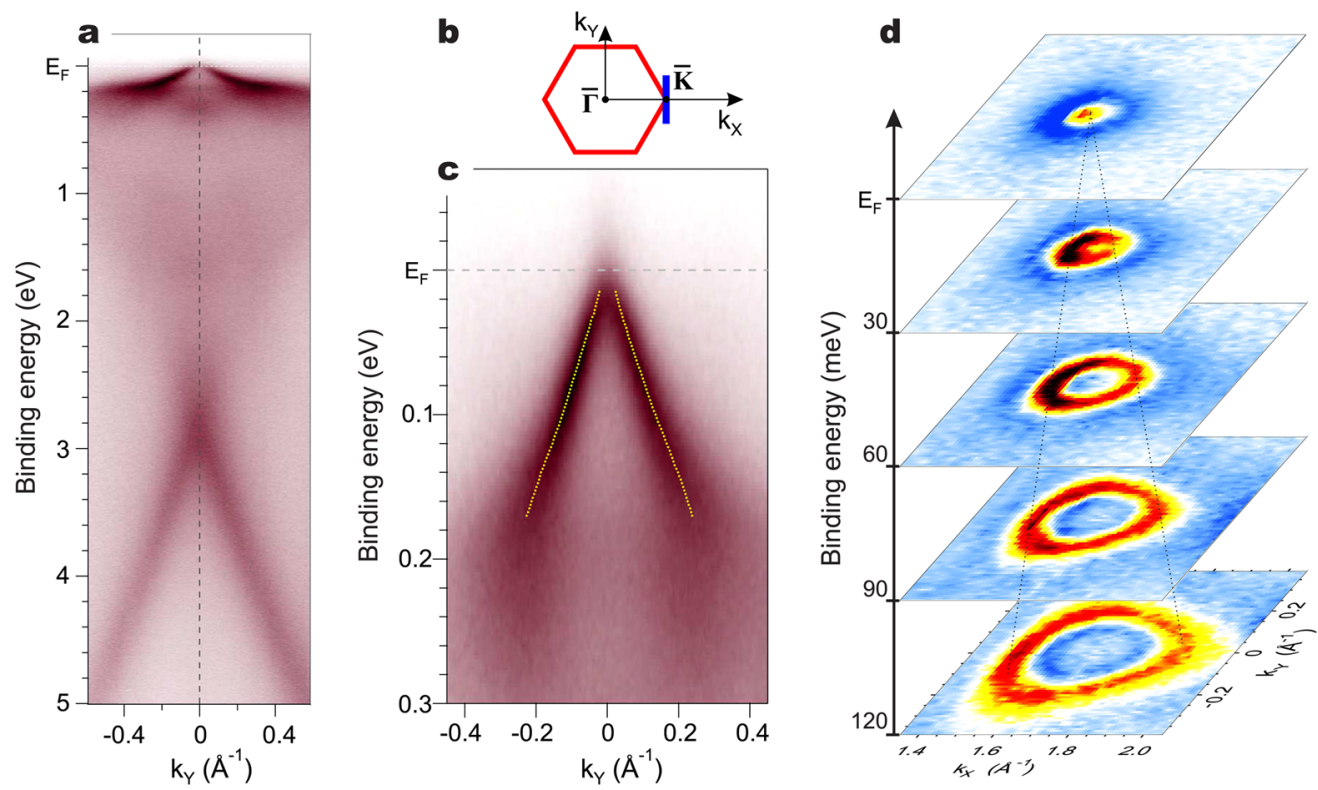

Figure 2. ARPES insight into the electronic structure of graphene/Co interface. (a) ARPES data taken near the $\overline{\mathbf{K}}$-point from well-oriented graphene on $\mathrm{Co}(0001)$ at room temperature, using $40 \mathrm{eV}$ photons with $\mathrm{s}+\mathrm{p}$ polarization. (b) Structure of graphene in momentum space; the line schematically indicates the direction of measurements. (c) ARPES insight into the minicone at $E_{\mathrm{F}}$ obtained with $28 \mathrm{eV}$ photons. (d) ARPES constant-energy maps of the minicone.

Looking at the ARPES-derived band maps, two interesting features may be seen: (i) the main Dirac cone, ${ }^{14}$ which is shifted to $\sim 2.8 \mathrm{eV}$ below $E_{\mathrm{F}}$, and (ii) another conelike feature near $E_{\mathrm{F}}$. The latter is shown magnified in Figure $2 \mathrm{c}$, where the Fermi level approaching is clearly visible. The dispersion of this state does not depend on the photon energy and its photoemission intensity is relatively high in the range of $h \nu=$ 22-60 eV. To prove this spectral feature has an intrinsic conical shape, representing carriers with the properties of Dirac Fermions, we took several constant energy maps. These are joined together in Figure 2d. Obviously, the ARPES data reveal trigonally warped contours of the discussed "minicone" feature, demonstrating certain similarity to the Dirac cone of quasifreestanding graphene. ${ }^{10}$ The photoemission intensity distribution is not symmetric, demonstrating higher intensity in the first BZ. However, the asymmetry is not so pronounced as in the case of main Dirac cone (see Figure S4 in Supporting Information). This difference can be explained by a significant contribution of cobalt d-orbitals to the conelike interface state. It should be noted that the dotted lines in Figure $2 \mathrm{c}$ show the result of fitting of momentum distribution curves with two Lorentzian peak functions. These lines show the band dispersion, which appears almost linear in the energy range of $30-150 \mathrm{meV}$; however the fit result is not well-defined at the $\overline{\mathbf{K}}$-point, where the two peaks become strongly overlapped. Thus, the exact shape of the cone apex cannot be reliably determined from experimental data. However, we can definitely conclude that the energy distance between the band and the Fermi level is smaller than the energy of thermal excitations at the room temperature $(26 \mathrm{meV})$.

As we see, the minicone feature is localized within a narrow energy region, $\sim 0.2 \mathrm{eV}$ below the $E_{\mathrm{F}}$. The slope of the linear part of the band, which is often identified with a Fermi velocity $v_{\mathrm{F}}$, is nearly ten times smaller than that observed in a freestanding graphene. The reduced $v_{\mathrm{F}}$ in the graphene/Co(0001) system reflects a certain influence of the "heavy"d-electrons of the cobalt substrate. This suggests that the minicone contains notable contribution of $3 d$ states of cobalt hybridized with $2 p$ states of carbon.

The Nature of the Mini Dirac Cone. For deeper understanding of the observed band structure we carried out $\mathrm{ab}$ initio calculations. Taking into account sublattice asymmetry, observed in STM images, we considered a top-fcc adsorption geometry, which we have found to be slightly more favorable energetically (by only $6 \mathrm{meV}$ per $\mathrm{C}$ atom) than the top-hcp structure. The ferromagnetic nature of Co results in exchange splitting of all the states within the graphene/ $\mathrm{Co}(0001)$ system and formation of the majority and minority states. When the freestanding graphene starts to interact with the Co substrate, the spin-up and spin-down states of the initially degenerate Dirac cone (dashed line in Figure 3a) are coupled differently with the respective spin-polarized Co states. The apex of the unperturbed Dirac cone falls directly into the local energy gap of the bulk majority states of Co. Therefore, the conical dispersion of spin-up states is mainly preserved near the cone apex. The interplay with cobalt bands only leads to the opening of a small gap of $\sim 0.33 \mathrm{eV}$ at the $E_{\mathrm{F}}$ and reducing the velocity of quasiparticles. This scenario explains the minicone formation (see Figure S1 in Supporting Information). The spindown Dirac cone apex strongly overlaps with the bulk projected Co minority states near $E_{\mathrm{F}}$, and their interaction pushes the lower cone apex down, leading to a huge splitting of $\sim 4.5 \mathrm{eV}$ in the $\pi$-band. It should be noted that in ref 14 the formation of a second Dirac cone was described theoretically and discussed in detail for the case of graphene/ $\mathrm{Ni}(111)$ system. It was emphasized that the second cone at the $E_{\mathrm{F}}$ is a counterpart of the main Dirac cone with its apex at $2.8 \mathrm{eV}$.

The minicone states are formed by the mixture of $\mathrm{C} 2 \mathrm{p}_{z}$ and Co $3 \mathrm{~d}$ states. They have an intrinsically two-dimensional nature and their wave functions are spatially located at the interface (see Figure S2 in Supporting Information). This theoretical finding is conclusively confirmed by the absence of the $k_{z}$ dispersion of the minicone feature in ARPES spectra. The minicone appears only in the majority spin bands, and therefore 

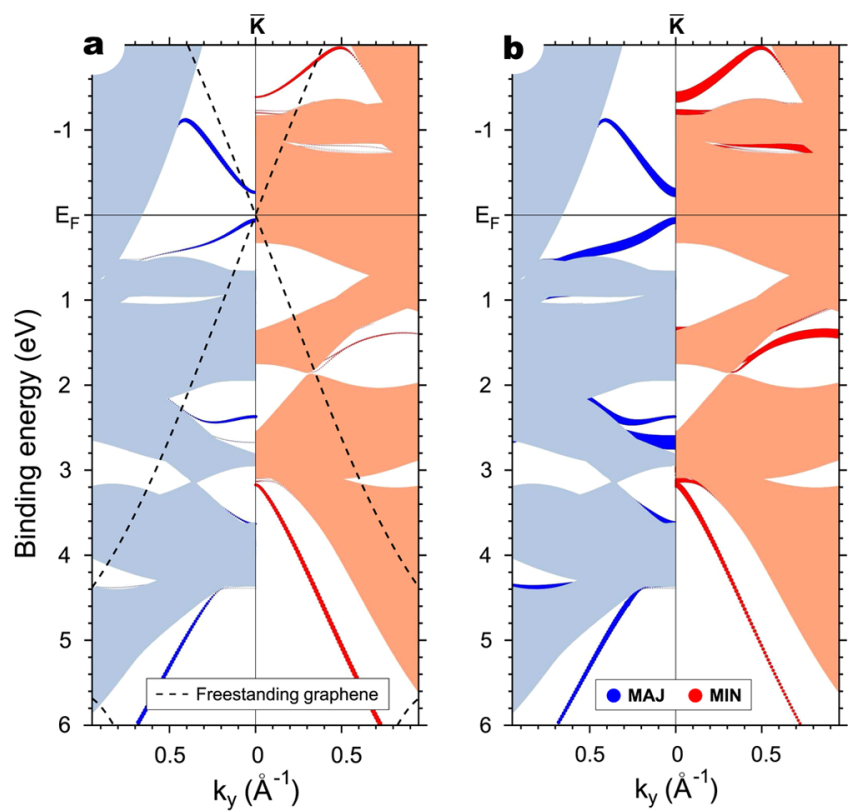

Figure 3. Theoretical insight into surface and bulk spin-resolved band structure of graphene/ $\mathrm{Co}(0001)$. Size of spots is proportional to the contribution of carbon (a) and upper cobalt layer atoms (b) to the wave function. The dashed line shows the bands of freestanding graphene. Shaded bands correspond to projected bulk Co states. The path in the k-space corresponds to the experiment geometry, shown in Figure $2 b$.

it can be considered as a $100 \%$ spin-polarized interface state. It should be noted that the ARPES data revealed an extensive region of linearity of the minicone dispersion, therefore it may be considered as a Dirac cone. The calculation demonstrates that the cone apex is parabolic due to presence of a gap. It implies that Dirac Fermions are not massless. The gap originates from graphene symmetry breaking in the top-fcc configuration and may vanish in a more symmetric bridge-top structure, as it was predicted for the graphene/ $\mathrm{Ni}(111)$ system. ${ }^{25}$ Because the upper unoccupied part of the minicone is not accessible with ARPES, we are not able to determine reliably which adsorption geometry was realized in experiment.

The predicted strong spin polarization of the minicone is experimentally confirmed by the SR-ARPES data shown in Figure 4 . The spectrum was taken along a cut through the $\overline{\mathbf{K}}$ -

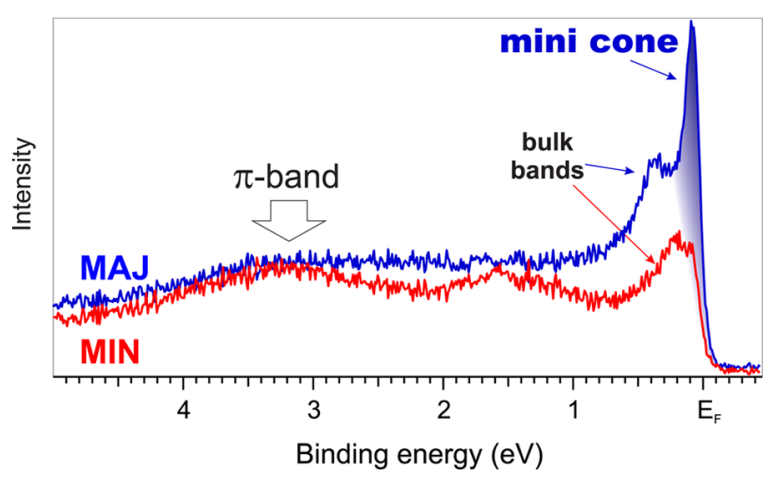

Figure 4. Spin-resolved ARPES view of the minicone feature. The spectra were taken at the $\overline{\mathbf{K}}$-point of the $\mathrm{BZ}$ along the vertical dashed line in Figure 2a. Momentum resolution was $\sim 0.2 \AA^{-1}, h \nu=37 \mathrm{eV}$. The sample was magnetized along the $\overline{\boldsymbol{\Gamma}} \overline{\mathbf{K}}$ direction. Magnetization along the $\overline{\boldsymbol{\Gamma}} \overline{\mathbf{M}}$ direction results in the similar SR-ARPES spectra. point. The majority spin channel exhibits a strong peak just below $E_{\mathrm{F}}$, while no peak is present in the minority spin signal. The peak in the majority channel reflects the highly spinpolarized minicone located in the spin-projected band gap of the Co substrate. Two bumps seen at 0.2 and $0.35 \mathrm{eV}$ in the minority and majority spectra, respectively, we attribute to the edges of bulk cobalt bands with high density of states. This is consistent with our calculation within the accuracy of DFT. A broad peak in the minority-spin signal at $\sim 3.2 \mathrm{eV}$ is related to the spin-polarized apex of the main Dirac cone, which is in agreement with the results of our calculation. The peak is broadened and shifted to higher binding energies (with respect to the data shown in Figure 2a) due to lower momentum resolution in spin-resolved mode.

In summary, the presented data clearly demonstrate the existence of a single-spin mini Dirac cone at $E_{\mathrm{F}}$ when the graphene monolayer is perfectly oriented on a $\mathrm{Co}(0001)$ surface. It is important to emphasize that those graphene/ $\mathrm{Co}(0001)$ samples that mainly consist of misoriented domains DO NOT reveal this minicone feature (see Supporting Information Figure S3b). The intensity of the minicone is proportional to the fraction of domains with $(1 \times 1)$ structure, which we believe is why the minicone was not detected and described in the previous studies. It should be noted that according to calculations, the band structure of the graphene/ $\mathrm{Ni}(111)$ interface is very similar to that of graphene/Co(0001). A spin-polarized minicone at the $E_{\mathrm{F}}$ was predicted for this system, too. ${ }^{14}$ It can be identified in the calculated band structure of graphene $/ \mathrm{Ni}(111)$ system. ${ }^{7,14,16,24,25}$ The epitaxial properties of the Ni-based system are more favorable, and the $(1 \times 1)$ structure is usually formed due to a perfect match of the lattice constants. Nevertheless, the experimental observation of a minicone in this system has been never reported. We have carefully measured ARPES spectra of graphene $/ \mathrm{Ni}(111)$ interface and found that the minicone appears in this system as well (see Figure S5 in Supporting Information). However, the feature is not as striking and pronounced as is seen in the case of the Co-system. It seems from the data that the minicone apex is located below the $E_{\mathrm{F}}$ and its upper part is visible, implying that the gap is very small $(<40 \mathrm{meV})$ or absent. According to the recent calculations ${ }^{25}$ such gap corresponds to a bridge-top adsorption geometry in which the upper metal layer does not break the symmetry of the graphene lattice. In the symmetry-breaking configurations like top-fcc or top-hcp, the gap is expected to reach $0.5 \mathrm{eV}^{25}$ This may indicate that in our sample the bridge-top geometry was dominating. However, the data in Supporting Information Figure S5 must be interpreted with a great caution, because the minicone falls into the region with high density of $\mathrm{Ni} 3 \mathrm{~d}$ minority states, which also contribute to the ARPES intensity and may mask the real dispersion of the interface state. Our finding together with the results of band structure calculations for multiple strongly bound graphene systems ${ }^{7,14,16,24,25,28}$ implies that the minicone formation is a quite general feature of graphene interfacing with d-metals.

The contact of graphene with a ferromagnetic material like $\mathrm{Co}$ or $\mathrm{Ni}$ can be a source of spin-polarized electrons and, therefore, is a very promising system for spintronic devices. ${ }^{4,6,11,16,29,30}$ Our results clearly demonstrate that the use of an epitaxial graphene/Co interface might be more efficient than the use of polycrystalline metal electrodes. This system has a unique electronic structure near the Fermi level-a single-spin interface state at the $\overline{\mathbf{K}}$-point of the BZ. The notable 
contribution of graphene $\pi$-orbitals to this state accounts for a good coupling between the wave function of spin and charge carriers at the interface and the wave function in freestanding graphene when it is used as a conducting channel between electrodes. We believe that this can provide good conditions for effective spin-polarized transport, which is in agreement with theoretical predictions. ${ }^{30}$

Methods. The clean $\mathrm{Co}(0001)$ surface was obtained by growing the crystalline cobalt film with a thickness of $10 \mathrm{~nm}$ on the clean $\mathrm{W}(110)$ substrate in ultrahigh vacuum (UHV) at a deposition rate of $1.5 \AA / \mathrm{min}$ at the room temperature. Graphene film was synthesized in the UHV chamber using chemical vapor deposition (CVD) by exposing the heated sample surface to propylene at a pressure of $10^{-6} \mathrm{mbar}$ and a temperature of $560-660{ }^{\circ} \mathrm{C}$ for $15 \mathrm{~min}$. At these conditions, the synthesis reaction is self-limiting. The growth starts immediately on a hot catalytically active metal surface and proceeds until the surface is covered by a single graphene layer. ${ }^{11,14,22}$ No further growth is possible on such graphenepassivated surface, therefore the synthesis always results in the formation of a complete graphene single monolayer. Monolayer graphene thickness was proved by X-ray photoemission spectroscopy using the intensity ratio of measured $\mathrm{C} 1 \mathrm{~s}$ and $\mathrm{Co}(\mathrm{Ni}) 3 \mathrm{p}$ lines. Depending on the synthesis temperature, different ordering of graphene domains was obtained. A similar procedure was used to form graphene on the $\mathrm{Ni}(111)$ surface. $^{31,32}$

Spin- and angle-resolved photoemission spectroscopy (SARPES and ARPES) was performed at the UE-112 PGM-1 beamline at BESSY II synchrotron radiation facility using RGBL-2 station. All measurements were done using linearly polarized radiation. Spin-resolved spectra were obtained from the data acquired in two opposite sample magnetization directions in order to eliminate spectrometer asymmetry. ${ }^{33}$ No smoothing was applied to the data.

Scanning tunneling microscopy (STM) images were obtained using Omicron VT SPM in the research resource center "Physical Methods of Surface Investigation" of St. Petersburg State University (SPbSU), where perfectly oriented graphene was synthesized on $\mathrm{Co}(0001)$ for the first time. The hexagonal close-packed crystal structure of cobalt film was confirmed after graphene synthesis using Bruker "D8 DISCOVER" X-ray diffractometer in the resource center for $\mathrm{X}$-ray Diffraction Studies of SPbSU.

We carried out ab initio electronic structure calculations of graphene/Co(0001) using the projector augmented-wave method $^{34}$ as implemented in the VASP code. ${ }^{35,36}$ The generalized gradient approximation to the exchange-correlation potential in the revised Perdew-Burke-Ernzerhof version $(\text { PBEsol) })^{37}$ was employed.

The system was simulated using the 19-layer-thick Co film with a graphene monolayer attached to both its sides. The lateral lattice parameter was fixed as in the experiment for bulk Co, $a=2.507 \AA$. All the atomic layers in the supercell, including graphene ones, were relaxed until the forces on each atom were less than $10^{-2} \mathrm{eV} / \AA$. The energy cutoff for the plane-wave expansion of wave functions was set to $400 \mathrm{eV}$, and a k-point grid of $12 \times 12 \times 1$ was used to sample the two-dimensional Brillouin zone.

\section{ASSOCIATED CONTENT}

\section{S Supporting Information}

Detailed results of the DFT calculations of graphene/Co(0001) system and SR-ARPES data for graphene/ $\mathrm{Ni}(111)$ system are presented. This material is available free of charge via the Internet at http://pubs.acs.org.

\section{AUTHOR INFORMATION}

\section{Corresponding Author}

*E-mail: usachov.d@gmail.com.

\section{Notes}

The authors declare no competing financial interest.

\section{ACKNOWLEDGMENTS}

The authors acknowledge Saint-Petersburg State University for a research Grants 11.37.634.2013 and 11.50.202.2015, RFBR (Grant 14-02-31150), and BMBF (Grant 05K12OD3). A.G. acknowledges support from the ERC Grant Super2D. M.M.O. and E.V.C acknowledge the financial support of the University of Basque Country UPV/EHU (Grant GIC07-IT-756-13) as well as of the Departamento de Educacion del Gobierno Vasco and the Spanish Ministerio de Ciencia e Innovacion (Grant FIS2010-19609-C02-01). We acknowledge Helmholtz Zentrum Berlin für Materialien und Energie for support within bilateral Russian-German Laboratory program. We cordially thank Dr. Allison L. Stelling for editing the manuscript.

\section{REFERENCES}

(1) Neto, A. H. C.; Guinea, F.; Peres, N. M. R.; Novoselov, K. S.; Geim, A. K. Rev. Mod. Phys. 2008, 81, 109-162.

(2) Das Sarma, S.; Adam, S.; Hwang, E. H.; Rossi, E. Rev. Mod. Phys. 2011, 83, 407-470.

(3) Avouris, P.; Dimitrakopoulos, C. Mater. Today 2012, 15, 86-97.

(4) Hill, E. W.; Novoselov, A. K.; Schedin, K.; Blake, P. IEEE Trans. Magn. 2006, 42, 2694-2696.

(5) Tombros, N.; Jozsa, C.; Popinciuc, M.; Jonkman, H. T.; van Wees, B. J. Nature 2007, 448, 571-U4.

(6) Karpan, V. M.; Giovannetti, G.; Khomyakov, P. A.; Talanana, M.; Starikov, A. A.; Zwierzycki, M.; van den Brink, J.; Brocks, G.; Kelly, P. J. Phys. Rev. Lett. 2007, 99, 176602.

(7) Khomyakov, P. A.; Giovannetti, G.; Rusu, P. C.; Brocks, G.; van den Brink, J.; Kelly, P. J. Phys. Rev. B 2009, 79, 195425.

(8) Nagashima, A.; Tejima, N.; Oshima, C. Phys. Rev. B 1994, 50, 17487-17495.

(9) Dedkov, Y. S.; Shikin, A. M.; Adamchuk, V. K.; Molodtsov, S. L.; Laubschat, C.; Bauer, A.; Kaindl, G. Phys. Rev. B 2001, 64, 035405.

(10) Varykhalov, A.; Sánchez-Barriga, J.; Shikin, A. M.; Biswas, C.; Vescovo, E.; Rybkin, A.; Marchenko, D.; Rader, O. Phys. Rev. Lett. 2008, 101, 157601.

(11) Varykhalov, A.; Rader, O. Phys. Rev. B 2009, 80, 035437.

(12) Brugger, T.; Günther, S.; Wang, B.; Dil, J. H.; Bocquet, M.-L.; Osterwalder, J.; Wintterlin, J.; Greber, T. Phys. Rev. B 2009, 79, 045407.

(13) Sánchez-Barriga, J.; Varykhalov, A.; Scholz, M.; Rader, O.; Marchenko, D.; Rybkin, A.; Shikin, A.; Vescovo, E. Diamond Relat. Mater. 2010, 19, 734-741.

(14) Varykhalov, A.; Marchenko, D.; Sánchez-Barriga, J.; Scholz, M. R.; Verberck, B.; Trauzettel, B.; Wehling, T. O.; Carbone, C.; Rader, O. Phys. Rev. X 2012, 2, 041017.

(15) Voloshina, E.; Dedkov, Y. Phys. Chem. Chem. Phys. 2012, 14, $13502-13514$.

(16) Generalov, A.; Voloshina, E.; Dedkov, Y. Appl. Surf. Sci. 2013, $267,8-11$. 
(17) Papagno, M.; Moras, P.; Sheverdyaeva, P. M.; Doppler, J.; Garhofer, A.; Mittendorfer, F.; Redinger, J.; Carbone, C. Phys. Rev. B 2013, 88, 235430.

(18) Pletikosić, I.; Kralj, M.; Pervan, P.; Brako, R.; Coraux, J.; N'Diaye, A. T.; Busse, C.; Michely, T. Phys. Rev. Lett. 2009, 102, 056808.

(19) Sutter, P.; Sadowski, J. T.; Sutter, E. Phys. Rev. B 2009, 80, 245411.

(20) Starodub, E.; Bostwick, A.; Moreschini, L.; Nie, S.; Gabaly, F. E.; McCarty, K. F.; Rotenberg, E. Phys. Rev. B 2011, 83, 125428.

(21) Rybkina, A. A.; Rybkin, A. G.; Fedorov, A. V.; Usachov, D. Y.; Yachmenev, M. E.; Marchenko, D. E.; Vilkov, O. Y.; Nelyubov, A. V.; Adamchuk, V. K.; Shikin, A. M. Surf. Sci. 2013, 609, 7-17.

(22) Vilkov, O.; Fedorov, A.; Usachov, D.; Yashina, L. V.; Generalov, A. V.; Borygina, K.; Verbitskiy, N. I.; Grüneis, A.; Vyalikh, D. V. Sci. Rep. 2013, 3, 2168.

(23) Eom, D.; Prezzi, D.; Rim, K. T.; Zhou, H.; Lefenfeld, M.; Xiao, S.; Nuckolls, C.; Hybertsen, M. S.; Heinz, T. F.; Flynn, G. W. Nano Lett. 2009, 9, 2844-2848.

(24) Garcia-Lekue, A.; Balashov, T.; Olle, M.; Ceballos, G.; Arnau, A.; Gambardella, P.; Sanchez-Portal, D.; Mugarza, A. Phys. Rev. Lett. 2014, 112, 066802.

(25) Zhang, W.-B.; Chen, C.; Tang, P.-Y. J. Chem. Phys. 2014, 141, 044708.

(26) Pacilé, D.; Lisi, S.; Di Bernardo, I.; Papagno, M.; Ferrari, L.; Pisarra, M.; Caputo, M.; Mahatha, S. K.; Sheverdyaeva, P. M.; Moras, P.; Lacovig, P.; Lizzit, S.; Baraldi, A.; Betti, M. G.; Carbone, C. Phys. Rev. B 2014, 90, 195446.

(27) Jacobson, P.; Stöger, B.; Garhofer, A.; Parkinson, G. S.; Schmid, M.; Caudillo, R.; Mittendorfer, F.; Redinger, J.; Diebold, U. J. Phys. Chem. Lett. 2012, 3, 136-139.

(28) Li, Y.; Chen, P.; Zhou, G.; Li, J.; Wu, J.; Gu, B.-L.; Zhang, S. B.; Duan, W. Phys. Rev. Lett. 2012, 109, 206802.

(29) Dedkov, Y. S.; Fonin, M.; Laubschat, C. Appl. Phys. Lett. 2008, 92, 052506.

(30) Cho, Y.; Choi, Y. C.; Kim, K. S. J. Phys. Chem. C 2011, 115, 6019-6023.

(31) Oshima, C.; Nagashima, A. J. Phys. Condens. Matter 1997, 9, 120.

(32) Grüneis, A.; Kummer, K.; Vyalikh, D. V. New J. Phys. 2009, 11, 073050 .

(33) Johnson, P. Rep. Prog. Phys. 1997, 60, 1217-1304.

(34) Blöchl, P. E. Phys. Rev. B 1994, 50, 17953-17979.

(35) Kresse, G.; Furthmüller, J. Phys. Rev. B 1996, 54, 11169-11186.

(36) Kresse, G.; Joubert, D. Phys. Rev. B 1999, 59, 1758-1775.

(37) Perdew, J. P.; Ruzsinszky, A.; Csonka, G. I.; Vydrov, O. A.; Scuseria, G. E.; Constantin, L. A.; Zhou, X.; Burke, K. Phys. Rev. Lett. 2008, 100, 136406. 